\title{
TREATMENT POSSIBILITIES FOR ACQUIRED AND HEREDITARY DISEASES BY GENE THERAPY: A REVIEW
}

\author{
P. V. KAMALA KUMARI ${ }^{*}$, G. EKSHITHA, V. HARIKA
}

Vignan Institute of Pharmaceutical Technology, Beside VSEZ, Duvvada, Visakhapatnam 530049

Email: kamalaparavastu@gmail.com

Received: 14 Oct 2020, Revised and Accepted: 19 Dec 2020

\begin{abstract}
Therapeutic nucleic acids demand specificity and accuracy in design as well as delivery strategies used in replacement or silencing of the target gene. Gene therapy is believed to be the therapy in which the root cause of the diseases can be treated at the molecular level. Generally gene therapy helps in the identification of the origin of the disorder instead of using drugs to diminish or control the symptoms. The application of nucleic acids to treat and control diseases is known as "gene therapy." Gene therapy consists on the substitution or addition of a functional gene into the nucleus of a living cell, in order to treat a disease or repair a dysfunction, caused by this gene failure. This therapy is used to correct defective genes, which are responsible for genetic diseases. Thus, gene therapy can be used to prevent, treat or regulate hereditary or acquired disorders, by the production of therapeutic proteins. The gene therapy is mediated by the use of viral and non-viral vectors to transport foreign genes into somatic cells to restorative defective genes. This review focuses on viral vectors in detail.
\end{abstract}

Keywords: Barriers, Viral vectors, Antisense oligonucleotide, Chimeric vectors

(C) 2021 The Authors. Published by Innovare Academic Sciences Pvt Ltd. This is an open access article under the CC BY license (https://creativecommons.org/licenses/by/4.0/) DOI: https://dx.doi.org/10.22159/ijap.2021v13i2.39995. Journal homepage: https://innovareacademics.in/journals/index.php/ijap

\section{INTRODUCTION}

Nucleic acids (NA) therapies have been gaining significant scientific and clinical interest over the last two decades. The main reasons for the failure are the occurrence of unexpected severe adverse effects during clinical trials, and the difficulties to accomplish a successful administration of deoxyribonucleic acid (DNA) and ribonucleic acid (RNA) molecules within the human body, related to the difficulties of targeting the cell's nucleus. Currently, several gene therapy medicines are approved for clinical use, especially in severe diseases (e. g., cancer and AIDS), where the benefit-risk balance accepted for therapeutic is small, compared to other less serious diseases.

Basically, NA therapy consists of two parts: first, the therapeutic NA itself, with plasmid DNA (pDNA) and different types of small RNA, and second, the carrier which delivers the NA to the desired site, including viral and non-viral vectors [1]. Nucleic acids, such as pDNA, antisense oligonucleotide, and RNA molecules, have a great potential to be used as therapeutics for the treatment of various genetic and acquired diseases [2]. Several strategies are being explored for their enhanced gene transfer, including design of different synthetic carriers [3] or the use of physical approaches [4]. Gene therapy emerged as the gold standard when two Nobel prizes were acclaimed by Andrew Fire and Craig Mello, who demonstrated the mechanism and application of RNA interference (RNAi) in 2006 [5]. Basically, gene therapy treatment involves three steps [6] as shown in fig. 1.

Furthermore, for a successful gene therapy process, an appropriate amount of the therapeutic gene (i.e. the transgene) must be delivered into the target tissue or cell, without exerting significant toxicity. Generally, two categories of gene therapy have emerged, the germ line and somatic gene therapy. The former consists in transferring the functional gene into germ cells, which means that it will be integrated in the individual genome, passing through generations. In the somatic gene therapy, the functional gene is introduced only in a specific target cell's (somatic cell's), without transmission of hereditary to the next generations. Currently, gene therapy offers new treatment possibilities for both acquired and hereditary diseases where conventional clinical procedures are less effective, such as cystic fibrosis, cancer, arthritis, peripheral vascular diseases, neurodegenerative disorders and AIDS [7]. Hereditary diseases are generally caused by deletion or mutation of a single cell, whereas in acquired diseases, a single gene cannot be defined as the only cause of disease [8].

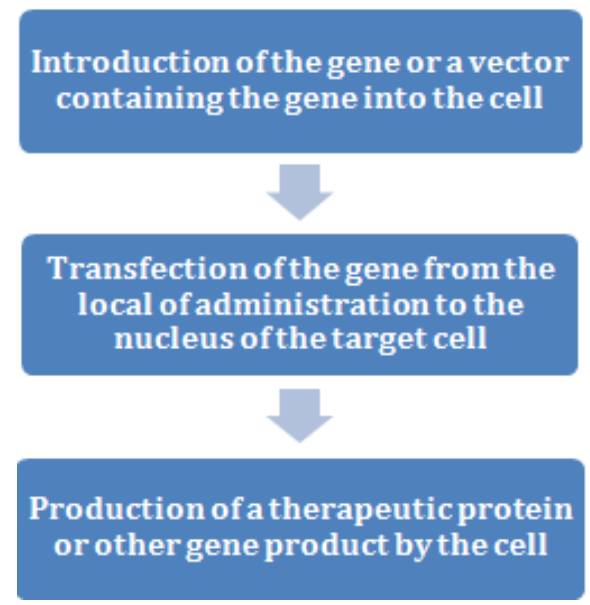

Fig. 1: Steps involved in gene therapy

Barriers to the nucleic acid delivery

In the context of efficient delivery of nucleic acids, it should fulfill two of the most important criteria, namely, safety and efficacy [9]. One of the challenges to the systemic delivery of nucleic acid therapeutics is the potential degradation of the NA by end nucleases in physiological fluids and the extracellular space [10]. Among all of the challenges in the delivery of nucleic acids, some important challenges are described and should be considered while designing the appropriate delivery approach (fig. 2).

\section{Intracellular bioavailability}

With the introduction of nucleic acids (naked or encapsulated form) in biological fluids faces many physiological or biological barriers leading to altered cellular bio distribution as well as overall intracellular bioavailability. Unmodified DNA and RNA, whether it may be a plasmid, siRNA, or shRNA, rapidly degrade in biological fluids by various extra-and intracellular enzymes before they can reach the surface of the target cells; this administration ultimately leads to the loss of therapeutic activity of nucleic acid [11] and poses a challenge to the effective delivery. 


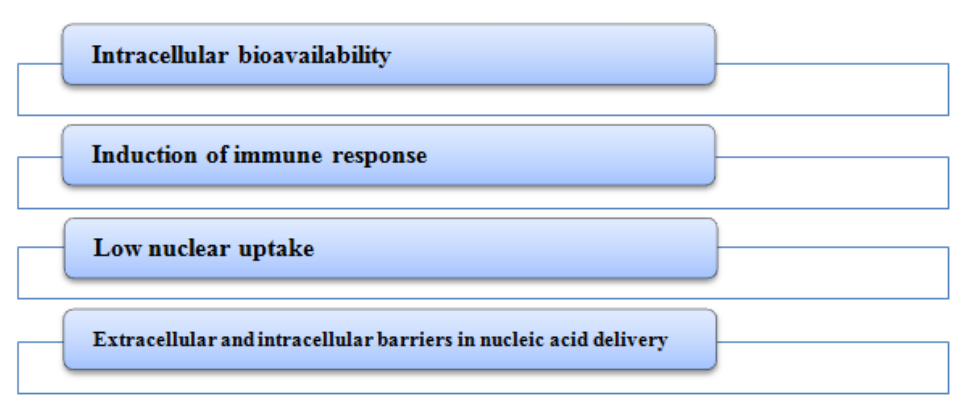

Fig. 2: Challenges while designing the appropriate delivery approach

\section{Induction of immune response}

The exogenously administered nucleic acid materials may trigger and activate the immune system, resulting in the release of inflammatory cytokines, which may lead to serious local and systemic inflammatory reactions [12]. The endosomal toll-like receptors (TLRs) are the most commonly identified mechanisms involved in the release of the pro-inflammatory cytokines. This immune mechanism can be augmented by the complexation of nucleic acid with cationic polymer or some lipids [13]

\section{Low nuclear uptake}

It is evident that there must be translocation between plasmid and nucleic acid for gene expression without considering the types of carrier and internalization pathways. Translocation, which involves both trafficking to the nucleus and penetration of the nuclear membrane through naked DNA or RNA into the nucleus, is usually ineffectual in both dividing and non-dividing cells. As per Bieber et al., (2002), only a small fraction of DNA/polyethyleneimine (PEI) complexes, which are internalized by the cells and released from the endosomes, successfully reaches inside the nucleus [14]. The characteristic of nuclear membrane permeability and the translocation process of the nucleus allow the import and export of specific molecules into and out of the nucleus [15] Due to the membrane pore size $(25 \mathrm{~nm})$, molecules smaller than $40 \mathrm{kDa}$ or complexes with a particle size of $25 \mathrm{~nm}$ can diffuse passively, whereas larger molecules cannot diffuse [16].

\section{Extracellular and intracellular barriers in nucleic acid delivery}

It is difficult to achieve the desired therapeutic effect of nucleic acid delivery by conventional delivery strategies due to several biological and cellular barriers (fig. 3 ) present in the body. Before reaching the targeted site, NAs face many obstacles to go across extracellular, cellular, and intracellular biological membranes [17]. Following systemic administration, these molecules have to go across various intracellular barriers such as intracellular trafficking, endosomal escape, and extracellular barriers like lipid bilayer membrane and various endothelial barriers [18]

The next barrier is the lack of techniques to study the intracellular trafficking of polyplexes to probe and elucidate steps that limit transfection efficiency [19].

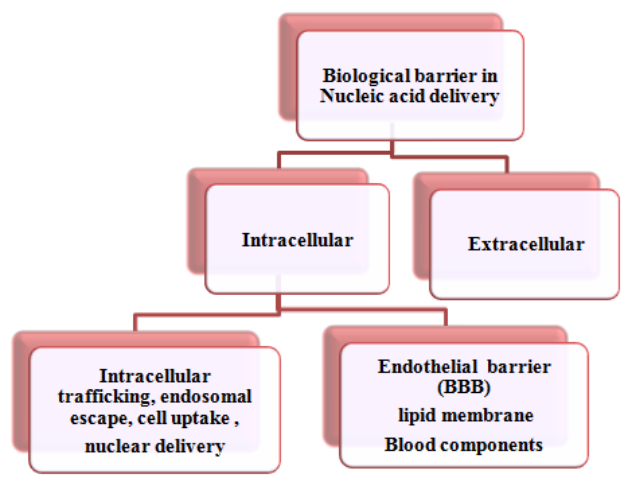

Fig. 3: Different types of biological barriers in nucleic acid delivery
Usually, the gene therapy is mediated by the use of viral and nonviral vectors to transport foreign genes into somatic cells to restorative defective genes [20]. Antisense oligonucleotide (AON), siRNA, and pDNA are the important types of nucleic acids for gene therapy which are principally utilized as potent therapeutic agents to silence the expressions of defective genes. Following paragraphs details of all three important types of nucleic acid delivery tools.

\section{Plasmid DNA}

Plasmid is a unique type of double-stranded DNA, usually circular in shape, used in gene therapy, and it contains the transgene that encodes for a specific protein. The size of the most commonly used pDNA ranges from a few hundred base pairs (bp) to several thousand bp [21]. Therapeutically, plasmids can be used for the treatment of several diseases, as well as an active constituent for vaccination. Promoter and enhancer sequences in pDNA, as regulatory signals, play an important role in regulating gene expression [22]. Promoter sequences are derived from viral origin such as cytomegalovirus and roux sarcoma virus, which initiate the transcription process by acting as a recognition site for the RNA polymerase which ensures high transcription efficiency. Enhancers are the regions in the pDNA that can be upstreamed or downstream from the promoter region. They act as binding sites for proteins that enhance the initiation of gene transcription. The selection of a suitable enhancer can improve transcription efficiency by several hundred folds $[23,24]$.

\section{Antisense oligonucleotide}

According to the idea of using block protein function as a drug molecular mechanism for treating diseases, antisense oligonucleotide therapy was developed. These oligonucleotides consist of small strands of DNA or RNA that can hybridize with specific DNA or mRNA (i.e. messenger RNA) sequences and suppress gene expression. The sequence of DNA or RNA that encodes the information for the protein production is called sense strand. In the double-strand DNA molecule, a complementary sequence is called antisense strand. Accordingly, it is possible to chemically synthesize antisense strands of nucleotides, which can bind with high level of affinity, i.e. selectively hybridize, to specific sense DNA or RNA strands, by Watson-Crick base-pairing rules. As a consequence, the process of DNA transcription to mRNA or the translation of mRNA to proteins is suppressed. Therefore, antisense oligonucleotides therapeutics can be used to block the expression of abnormal genes and have been investigated for different medical applications, such as oncologic, infectious and cardiovascular diseases.

\section{RNA interference}

RNA interference (RNAi) reveals great therapeutic potential as a post-translation gene regulation process for a variety of diseases, including genetic disorders, autoimmune diseases, viral infections, and cancer [25, 26].

\section{Types of RNAs}

\section{Small interfering $R N A$}

The small interfering RNA (siRNA) or silencing RNA is a doublestranded RNA molecule with $20-25$ bp that has a major role in RNAi pathways, where it interferes with the expression of specific genes with complementary nucleotide sequences [27]. RNA interference is a 
type of regulatory mechanism that most commonly occurs in the cytoplasm of most eukaryotic cells to control gene activity [28, 29]. In the process of RNA interference, long double-stranded RNA is cleaved into shorter double-stranded RNA segments (siRNA) by the dicer proteins. After cellular entry, the siRNA duplex is assembled into large protein assemblies, called the RNA-induced silencing complexes (RISC) where the sense strand of siRNA is removed and cleaved by the RNase H-like activity of Ago-2 (cleavage-dependent pathway, member of the argonaut family of proteins) [30] and is a major component of RISC [31]. The other members of Argonaut family, like Ago-1, Ago-3, and Ago-4, do not have specific endonuclease activity, and they function through a cleavage-independent pathway [32]

\section{Small hairpin RNA (shRNAs)}

shRNAs is a type of RNA consisting of 25 to 29 bases and a loop of 4-23 nucleotides sequence that can induce gene silencing through RNAi [33]. shRNA is processed in the cytoplasm by the dicer protein to remove the loop to produce siRNA, which then forms RISC followed by subsequent cleavage of the target mRNA [34]. Bifunctional shRNA employs two shRNA, one with the perfect match for cleavagedependent RISC loading and the second with mismatched sense strands (at the central location) for cleavage-independent RISC loading [35] has been designed to enhance the efficiency of shRNA.

\section{MicroRNA}

MicroRNA (miRNAs) are mature and short (20-24 nucleotides) single-stranded, non-coding RNAs that bind to partially complementary sites, called miRNA binding sites. miRNA silences gene expression by affecting the stability of target mRNAs or via translational repression. miRNAs are transcribed from DNA initially as long primary transcripts termed as "pri-miRNA" comprised of an RNA hairpin structure which is cleaved from the pre-miRNA by the nuclear microprocessor complex and the endonuclease drosha complex in the nucleus, which subsequently gives rise to precursor miRNA termed as "pre-miRNA" [36]. The pre-miRNA is transported from the nucleus to the cytoplasm by exportin-5 [37] and is further cleaved by the endonuclease dicer into short double-stranded RNA in which one strand is the mature miRNA and its counterpart is known as the miRNA [38].

\section{Vectors in nucleic acid delivery}

Vectors are the carriers that act as the delivering vehicles for the drugs, nucleic acid, and various biotechnological active substances. For delivering nucleic acids to the cells, these are broadly divided into two categories named viral and non-viral vectors. NA can be delivered to patients by either ex vivo or in vivo methods. In vivo methods involve vector delivery, through intravenous injection, or direct local implantation, to a specific tissue. In contrast, ex vivo methods comprise an initial removal of cells from the patient, followed by their in vitro genetic manipulation and subsequent reintroduction of modified cells to the patient [39]. Ex vivo therapy is considered to be safer and more effective than in vivo therapy because cells modified in vitro can be selected and amplified, before patient reinfusion. However, when target cells are difficult to collect or manipulate in vitro, the in vivo procedure remains the only suitable option [40].

\section{Viral vectors}

Viral vectors are the most efficient vehicles for NA transfer due to their ability to infect a large number of cells. Since viruses have evolved natural mechanisms to deliver their genetic material into cells, they are excellent vectors to deliver transgenes [41]. Briefly, viral vectors consist of viruses that are modified to be replication-incompetent but can deliver NA into the cells [42]. The use of viral vectors for NA delivery presents several disadvantages, such as $[43,44]$ :

- They might alter more cells than the intended ones;

- The transgene can be inserted in the wrong location of the DNA strand, causing damage;

- The gene can be over-expressed;

- The modified virus can cause body inflammation or trigger an immune reaction;
- The virus can be transmitted to other individuals or to the environment.

Apart from all these disadvantages, the majority of the viral vectors used in human clinical trials appear to be safe and well-tolerated by patients [44]

\section{Converting a virus into a vector}

In the life cycle of a virus, two distinct phases should be considered: infection and replication [45]. Viral vectors are parasitic particles, which are naturally capable of entering in the host cells and transferring to them their genetic sequences. Viruses become replication-incompetent by deletion of a portion of the genetic sequence essential for viral propagation and subsequent replacement of this sequence by a transgene [39]. The construction of a viral vector requires sufficient knowledge and an effective strategy. The viral life cycle and its genetic sequence must be well known in order to delete non-essential and virulent genes. The genome deletion enables the construction of an on-pathogenic vector, which is unable to infect the patient but maintains its replicative ability [46]. The viral genome comprises genes and cis-acting gene regulatory sequences. To produce a viral vector, the genes and cis-acting sequences should be separated into distinct NA molecules, to prevent their reconstitution in productive viral particles and to allow the maintenance of the efficacy and safety of the system [47]. As there are no universally applicable ideal viral vector available, several characteristics of each vector and type of disease need to be defined before making decisions about which vector type should be applied [48]. Considerations regarding viral vectors include the ability to attach and enter into the target cells, the ability to transfer the NA to the nucleus, the ability of NA being expressed in the nucleus for a prolonged period of time and the lack of toxicity [49]. Accordingly, an ideal NA delivery vector should have the following characteristics [50]:

- High specificity;

- Efficient NA delivering, in the amount needed for clinical application;

- Unrecognized by the immune system;

- Ability to be purified in high concentrations;

- Safe for the patient and also for the environment;

- Express the gene during the required period.

The purified vectors generally are stored either refrigerated or frozen, and display usable shelf lives of more than 2 y [51]. There are five main classes of viral vectors commonly used for NA delivery (fig. 4). These can be categorized in two groups, according to whether their genomes integrate into host cellular chromatin (retrovirus and lentiviruses), or persist in the cell nucleus mainly as extra-chromosomal episomes (adenovirus, adeno-associated virus and herpes simplex virus) [52] Besides these, many other viral vectors have been used for NA delivery (e. g. poxvirus, alphavirus, human cytomegalovirus) [44].

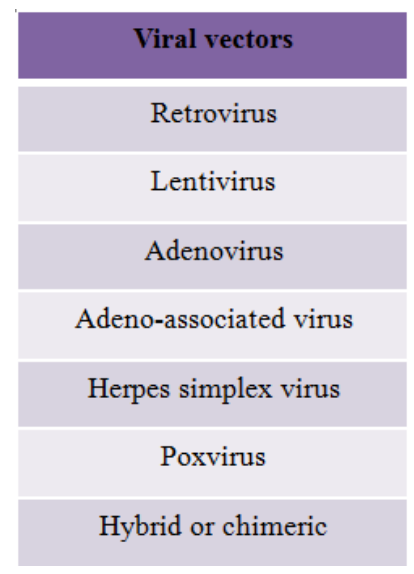

Fig. 4: Classification of viral vectors 


\section{Retrovirus}

Retrovirus are RNA-based vectors that replicate through a DNA intermediate and comprise both a capsid and an envelope protein containing a lipid bilayer. The most widely characterized retroviral vector is the Moloney murine leukemia virus (MoMuLV), which was the first used for the treatment of adenosine deaminase severe combined immunodeficiency (ADA-SCID) [53]. The more simple class of retroviruses contains three major coding segments gag (codes for core viral protein), pol (codes for reverse transcriptase) and env (codes for viral envelope proteins). At either end of the viral genome are the long terminal repeat (LTR) sequences, which harbor powerful promoter and enhancer regions and sequences required to promote integration into the host DNA [39, 51, 54].

Virus infection begins with the binding of the viral envelope glycoprotein to cell receptors. Within the cytoplasm, the viral reverse transcriptase enzyme converts the RNA genome into linear double-stranded DNA, which is randomly integrated into the host cell genome $[40,51]$. The main concerns in using recombinant retroviruses are the risk of accidental random integration into the host chromosome, resulting in deleterious effects, such as the activation of certain proto-oncogenes by insertional mutagenesis, or inactivation of other tumor suppressor genes [41]. Through recombinant DNA techniques, the endogenous viral genes gag, pol and env required for the reproduction of retrovirus genome are removed and replaced by the exogenous gene of interest. The advantages of using retroviral vectors include:

- Stable integration into the host genome;

- Efficient gene transfer by generating enough viral titre

- Ability to infect several different cell types;

- Capacity to transport foreign genes of reasonable sizes $(<8 \mathrm{~kb})$.

In contrast, this type of vectors also present some disadvantages [44, 45], including:

- Poor stability to transport NA;

- Risk of damage during purification and concentration process;

- Relatively low carrying capacity;

- Inability to infect non-dividing cells;

- Possible insertional mutagenesis.

\section{Lentivirus}

Lentivirus, such as human immunodeficiency virus type 1(HIV-1), are a subcategory of the retrovirus family, which are capable of infecting non-dividing cells [45]. This ability of lentivirus is due to their targeting the nucleus without the requirement of mitosis [44]. Despite all retrovirus have only three structural genes, the lentivirus have two additional genes (tat and rev) and four accessory genes (vif, vpu, nif, vpr). Although lentivirus can integrate in the host genome, their integration sites are significantly more restricted than for other retroviruses [56]. Lentivirus vectors were developed with the aim to become the substitutes of onco-retrovirus, due to their ability of transgene expression and enhanced safety [57]. These vectors have been successfully applied in gene therapy because they offer greater advantages over the other retrovirus counterparts [58]. Lentivirus vectors have the advantages of long-term transgene expression, low immunogenicity, and the ability to accommodate larger transgenes. Furthermore, due to their natural integration ability, lentivirus have a lot of potential for central nervous system gene delivery [59].

\section{Adenovirus}

Adenoviruses contain a linear, double-stranded DNA genome, around $36 \mathrm{~kb}$ in size, package within a non-enveloped icosahedral capsid. The adenoviral infection involves binding of the viral capsid proteins existing in specific receptors of host cells and subsequent internalization, by receptor-mediated endocytosis. However, unlike retroviruses, upon cellular infection, adenoviral DNA becomes localized in the nucleus as an episomal element but does not integrate the host cell genome, which means that there is a low risk of insertional mutagen city $[39,60]$.

The advantage of these viruses is their ability to infect target cells, which greatly minimizes the risk of insertional mutagenesis. However, the failure of the adenoviral-based DNA to integrate into the host cell generally means that its survival and, therefore, the duration of gene expression is limited [51]. Studies on the development of adenoviral vectors brought significant progress in the target cell specificity, long-term expression of the transgene, immunogenicity and in vivo toxicity [61].

Adenovirus gene delivery systems have been used more frequently for in vivo approaches than retrovirus-based systems. Although early generations of adenovirus showed toxic side effects and immune responses, newer second and third-generation vectors with many of the genes deleted have demonstrated significant improvements [48]. Adenovirus possesses strong tropism to epithelial tissues and was the first viral vectors tested for gene therapy of cystic fibrosis [62]. Adenoviral vectors have also been used for treating peripheral vascular diseases and coronary artery diseases [44, 47]. Moreover, apart from using adenovirus vectors as carriers for transgenes, these virus itself provides antitumor activity, by targeting tumor cells with the defective $\mathrm{p} 53$ protein [44].

\section{Adeno-associated virus}

Adeno-associated virus are small, non-enveloped, linear single stranded DNA virus, which belong to the Parvoviridae family. This virus is replication-defective and to replicate within a host cell nucleus needs a helper virus, such as adenovirus or herpes virus [49, 63]. These systems provide a mechanism of gene transfer into nondividing cells, and also seem to facilitate the long-term expression of the transferred genetic material. In contrast to adenovirus, the NA transferred by adeno-associated viruses appears to be integrated into the host cell genome [51].

Adeno-associated virus present some advantages over other viral vectors, since they are non-pathogenic, non-immunogenic, and are able to infect a wide variety of dividing and non-dividing cells [39, $44,49]$. Besides, adeno-associated virus are unique among virus that have been used for gene therapy, because although they are found in human population, they do not appear to be associated with any disease. The small size and simplicity of the vector particle enables the systematic administration of high doses of the vector, without eliciting acute inflammatory responses or toxic side effects [52]. However, the restricted tropism of adenoassociated virus and its relatively small capacity for insertion of transgenes (ranging from 4.1 to $4.9 \mathrm{~kb}$ ) are some examples of the limitations of this viral vector $[39,44,49]$. In contrast, it has been shown that these vectors are suitable for both in vitro and in vivo gene transfer into muscle, brain, neurons and liver cells [44].

\section{Herpes simplex virus}

Herpes simplex virus are a class of double-stranded DNA virus, characterized by a large genome ( $152 \mathrm{~kb})$, belonging to the family Herpes viridae, which have the ability to infect cells of the nervous system [45]. Herpes virus are very promising vehicles for gene transfer, because of their ability to persist, after a primary infection, in a state of latency where the disease is absent in human hosts with normal immune status [47]. These vectors are of interest mainly because of its large packaging capacity for transgenes, allowing for the inclusion of more than one transgene and also large transcriptional regulatory elements and its natural tropism toward neuronal cells.

Different aspects of the herpes virus make them attractive for using as vectors for gene therapy [64]: highly infectious capacity;

- Virus receptors are widely expressed on the surface of numerous cell types;

- Non-dividing cells may be efficiently infected and transduced;

- Recombinant virus can be easily produced to high titer and purity;

- The latent behavior of the virus may be exploited for stable longterm expression of therapeutic transgenes in neurons. 


\section{Poxvirus and other viral vectors}

A special emphasis should also be given to poxvirus, which are large complex enveloped viruses with an enveloped double-stranded DNA genome of approximately $190 \mathrm{kbp}$ in length. The orthopoxvirus subfamily includes vaccinia virus, which had a crucial role in one of the greatest achievements in medicine: the eradication of smallpox. One of the main advantages of these viruses is their high insertion capacity for multiple foreign genes. They have the ability to integrate more than $25 \mathrm{kbp}$ heterologous DNA [44, 65]. Numerous inherent biological properties of vaccinia and other poxvirus make them ideal to express foreign antigens and so they have been used in many vaccine trials [66] and in the treatment of cancers [67].

Recently, a new class of oncolytic poxvirus has been developed, which combines targeted and armed approaches for treating cancer. Initial preclinical and clinical results show that products from this therapeutic class can systemically target cancers through a highly selective mechanism of action [68].

Poxvirus has several advantages as oncolytic therapeutic viral vectors, including

- Safety in humans,

- Ease of production,

- Stability of virus preparations, and

- Feasibility of genetic manipulation for transgene expression

- Replication takes place entirely in the cytoplasm of an infected cell, and thereby viral genome integration into host chromosomes does not occur.

Four poxvirus; VV (Orthopoxvirus), MYXV (Leporipoxvirus), racoonpoxvírus (Orthopoxvirus), and yaba-like disease virus (Yata poxvirus), from three different genera have been investigated. All of these viruses undergo productive lytic replication in most human cancer cells. The most frequently used vectors are based on Semliki Forest virus (SFV), Sindbis virus (SIN) and Venezuelan Equine Encephalitis (VEE) virus. Among others, human cytomegalovirus, influenza virus, and foamy virus have also been used to engineer vectors with feasible insertion capacities, expression efficiencies and cell tropism of viral infection [44].

\section{Hybrid or chimeric vectors}

Viral vectors are effective to transport genetic material within the cells. However, clinical trials showed that these vectors have some inherent limitations for therapy, namely, immunogenicity, toxicity, limited loading capacity, and failure of long-term adequate transgene expression. To overcome these drawbacks, attempts have been made, in order to combine the best characteristics of different viruses, by means of hybrid vectors [69].

These systems are more complex than conventional viral vectors, because they have components of two or more viruses or are combined with other proteins or genetic components, with the following objectives $[40,69]$.

- To target specific tissues or cell types;

- To accommodate large transgenes and regulatory elements;

- To achieve stable and regulated transgene expression;

- To retain high transduction efficiency and titers while minimizing undesired toxicity or immune responses.

Adenovirus and adeno-associated virus have attractive and complementary properties that can be exploited for gene transfer purposes. Adenoviruses combined with adeno-associated virus allowed the construction of hybrids with site-specific gene integration properties [70]. The hybrid adenovirus-retrovirus vector is based on the addition of retroviral integration machinery into adenoviruses, which maintains the attributes of these viruses for in vivo gene delivery and of retroviruses for long-term transgene expression [71]. Herpes simplex-adeno-associated virus hybrid vectors also proved the capacity to extend transgene expression in non-dividing hepatocytes and neurons in vitro [72] and in vivo [73]. Vectors based on hybrids consisting of adeno-associated virus types 2 and 5 were evaluated for muscle-directed gene transfer. Hildinger et al. showed that these types of hybrid vectors may have a number of advantages for muscle gene therapy application [74].

\section{CONCLUSION}

The extracellular, intracellular and other barriers offer added hurdles in the delivery of DNA, RNA, and antisense oligonucleotides to the affected cells. Another challenge, which has an equal impact to the delivery of genes, is the unstable nature of the DNA under the influence of the enzymes DNAase and RNAase. Similar to drug delivery, the encapsulation and/or conjugation of genes to the polymeric carriers has always been advantageous in terms of the effective transfection and delivery to the targeted cells. The size has played a crucial role in this regard and the smaller the size, the more the chance of the vector to be bypassed from the mononuclear phagocytic system of the body, which would, in turn, increase its overall circulation time and stability. Gene delivery has emerged as a promising approach for the treatment of complex disorders such as cancer, Alzheimer's, AIDS and related disorders since 1996. Future studies may include the co-delivery of antibodies with the relevant DNA or RNA.

\section{ACKNOWLEDGEMENT}

The authors are thankful to the management of Vignan Group of Institutions for providing the necessary online facilities to carry out this review.

\section{FUNDING}

Nil

\section{AUTHORS CONTRIBUTIONS}

All the authors have contributed equally.

\section{CONFLICT OF INTERESTS}

\section{Declared none}

\section{REFERENCES}

1. Mesnil M, Yamasaki H. Bystander effect in herpes simplex virus-thymidine kinase/ganciclovir cancer gene therapy: role of gap junctional intercellular communication. Cancer Res 2000;60:3989-99.

2. Zhu L, Mahato RI. Lipid and polymeric carrier-mediated nucleic acid delivery. Expert Opin Drug Delivery 2010;7:1209-26.

3. Wolff JA, Malone RW, Williams P, Chong W, Acsadi G, Jani A. Direct gene transfer into mouse muscle in vivo. Science 1990;247:1465-8.

4. Gill HS, Prausnitz MR. Coated microneedles for transdermal delivery. J Controlled Release 2007;117:227-37.

5. Fire A, Xu S, Montgomery MK, Kostas SA, Driver SE, Mello CC. Potent and specific genetic interference by double-stranded RNA in Caenorhabditis elegans. Nature 1998;391:806-11.

6. Patil PM, Chaudhari PD, Megha S, Duragkar NJ. Review article on gene therapy. Int J Genetics 2012;4:74-9.

7. Al-Lawatia YMM, Mullaicharam AR. Viral vectors for gene therapy: a review. Res J Pharm Biol Chem Sci 2013;4:813-23.

8. Cevher E, Sezer AD, Calar E. In: Gene delivery systems: Recent progress in viral and non-viral therapy. Sezer AD. Ed. In tech: Croatia; 2012. p. 337-470.

9. Pathak A, Patnaik S, Gupta KC. Recent trends in non-viral vector-mediated gene delivery. Biotechnol J 2009;4:1559-72.

10. Yin H, Kanasty RL, Eltoukhy AA, Vegas AJ, Dorkin JR, Anderson DG. Non-viral vectors forgene-based therapy. Nat Rev Gene 2014;15:54155.

11. Harada Shiba M, Yamauchi K, Harada A, Takamisawa I, Shimokado K, Kataoka K. Polyioncomplex micelles as vectors in gene therapy-pharmacokinetics and in vivo gene transfer. Gene Ther 2002;9:407-14.

12. Kariko K, Bhuyan P, Capodici J, Weissman D. Small interfering RNAs mediate sequence-independent gene suppression and induce immune activation by signaling through tolllike receptor 3. J Immunol 2004;172:6545-9. 
13. Yoshida H, Nishikawa M, Yasuda S, Mizuno Y, Toyota H, Kiyota $\mathrm{T}$, et al. TLR9-dependent systemic interferon-beta production by intravenous injection of plasmid DNA/cationic liposome complex in mice. J Gene Med 2009;11:708-17.

14. Bieber T, Meissner W, Kostin S, Niemann A, Elsasser HP Intracellular route and transcriptional competence of polyethyleneimine-DNA complexes. J Controlled Release 2002;82:441-54

15. Liu G, Li D, Pasumarthy MK, Kowalczyk TH, Gedeon CR, Hyatt $\mathrm{SL}$, et al. Nanoparticles of compacted DNA transfect postmitotic cells. J Biol Chem 2003;278:32578-86.

16. Roth CM, Sundaram S. Engineering synthetic vectors for improved DNA delivery: insights from intracellular pathways. Annu Rev Biomed Eng 2004;6:397-426.

17. Zhu L, Lu Y, Miller DD, Mahato RI. Structural and formulation factors are influencing pyridinium lipid-based gene transfer. Bioconjugate Chem 2008;19:2499-512.

18. Belting M, Sandgren S, Wittrup A. Nuclear delivery of macromolecules: barriers and carriers. Adv Drug Delivery Rev 2005;57:505-27.

19. Read ML, Logan A, Seymour LW. Barriers to gene delivery using synthetic vectors. Adv Genet 2005;53:19-46.

20. Luo D, Saltzman WM. Synthetic DNA delivery systems. Nat Biotech 2000a; 18:33-7.

21. Yin $\mathrm{W}$, Xiang P, Li Q. Investigations of the effect of DNA size in transient transfection assay using dual luciferase system. Anal Biochem 2005;346:289-94.

22. Williams JA, Carnes AE, Hodgson CP. Plasmid DNA vaccine vector design impact on efficacy, safety and upstream production. Biotechnol Adv 2009;27:353-70.

23. Asoh S, Lee Kwon W, Mouradian MM, Nirenberg M. Selection of DNA clones with enhancer sequences. Proc Natl Acad Sci 1994;9:6982-6

24. Noss KR, Wolfe SA, Grimes SR. Upregulation of prostate-specific membrane antigen/folatehydrolase transcription by an enhancer. Gene 2002;285:247-56.

25. Williford MJ, Wu J, Ren Y, Archang MM, Leong KW, Mao HQ Recent advances innanoparticle-mediatedsiRNA delivery. Annu Rev Biomed Eng 2014;16:347-70.

26. Bumcrot D, Manoharan M, Koteliansky V, Sah DWY. RNA therapeutics: a potential new class of pharmaceutical drugs. Nat Chem Biol 2006;2:711-9.

27. Agrawal N, Dasaradhi PVN, Mohmmed A, Malhotra P, Bhatnagar RK, Mukherjee SK. RNA interference: biology, mechanism, and applications. Microbiol Mol Biol Rev 2003;67:657-85.

28. Fougerolles A, Vornlocher HP, Maraganore J, Lieberman J. Interfering with disease: a progressreport on siRNA-based therapeutics. Nat Rev Drug Discovery 2007;6:443-53.

29. Grimm D, Kay MA. Therapeutic application of RNAi: is mRNA targeting finally ready forprime time? J Clin Invest 2007; 117:3633-41

30. Preall JB, Sontheimer EJ. RNAi: RISC gets loaded. Cell 2005; 123:543-5.

31. Hammond SM, Boettcher S, Caudy AA, Kobayashi R, Hannon GJ. Argonaute2, a link between genetic and biochemical analyses of RNAi. Science 2001;93:1146-50

32. Watts JK, Deleavey GF, Damha MJ. Chemically modified siRNA tools and applications. Drug Discovery Today 2008;13:842-55.

33. Paddison PJ, Caudy AA, Bernstein E, Hannon GJ, Conklin DS, Short hairpin RNAs (shRNAs)induce sequence-specific silencing in mammalian cells. Genes Dev 2002;16:948-58.

34. Chen Y, Cheng G, Mahato RI. RNAi for treating hepatitis B viral infection. Pharm Res 2008;25:72-86.

35. Rao DD, Vorhies JS, Senzer N, Nemunaitis J. siRNA vs. shRNA similarities and differences. Adv Drug Delivery Rev 2009;61:746-59.

36. Lee Y, Jeon K, Lee JT, Kim S, Kim VN. MicroRNA maturation: stepwise processing and subcellular localization. EMBO I 2002;21:4663-70.

37. Zeng Y, Cullen BR. Structural requirements for pre-microRNA binding and nuclear export by exportin 5. Nucleic Acids Res 2004:32:4776-85

38. Yang M, Mattes J. Discovery, biology and therapeutic potential of RNA interference, micro-RNA and antagomirs. Pharmacol Ther 2008;117:94-104.
39. Phillips JE, Gersbach CA, Garcia AJ. Virus-based gene therapy strategies for bone regeneration. Biomaterials. 2007;28:211 29.

40. Huang S, Kamihira M. Development of hybrid viral vectors for gene therapy. Biotechnol Adv 2013;31:208-23.

41. Seth P. Vector-mediated cancer gene therapy: an overview. Cancer Biol Ther 2005;4:512-7.

42. Cevher E, Sezer AD, Caglar E. Gene delivery systems: recent progress in viral and non-viral therapy. Recent Adv Novel Drug Carrier Syst 2012:437-70. DOI:10.5772/53392

43. Akhtar N, Akram M, Asif HM, Usmanghani K, Shah SA, Rao SA, et al. Gene therapy: a review article. J Med Plants Res 2011;5:1812-7.

44. Walther W, Stein U. Viral vectors for gene transfer. Drugs 2000;60:249-71.

45. Mahato RI, Smith LC, Rolland A. Pharmaceutical perspectives of nonviral gene therapy. In: Advances in genetics. Academic Press 1999;41:95-156.

46. Levesque C. Vector-mediated gene therapy and the herpes simplex virus. BioTeach J 2004;2:112-6.

47. Kay MA, Glorioso JC, Naldini L. Viral vectors for gene therapy: the art of turning infectious agents into vehicles of therapeutics. Nat Med 2001;7:33-40.

48. Lundstrom K, Boulikas T. Viral and non-viral vectors in gene therapy: technology development and clinical trials. Technol Cancer Res Treat 2003;2:471-85.

49. Daya S, Berns KI. Gene therapy using adeno-associated virus vectors. Clin Microbiol Rev 2008;21:583-93.

50. Misra S. Human gene therapy: a brief overview of the genetic revolution. J Assoc Physicians India 2013;61:127-33.

51. Gad SC. editor. Handbook of pharmaceutical biotechnology. John Wiley and Sons; 2007.

52. Thomas CE, Ehrhardt A, Kay MA. Progress and problems with the use of viral vectors for gene therapy. Nat Rev Genetics 2003;4:346-58.

53. Crommelin DJ. Formulation of biotech products, including biopharmaceutical considerations. In: Pharmaceutical biotechnology. Springer, New York NY; 2013. p. 69-99.

54. Bleiziffer O, Eriksson E, Yao F, Horch RE, Kneser U. Gene transfer strategies in tissue engineering. J Cell Mol Med 2007;11:206-23

55. Stone D, David A, Bolognani F, Lowenstein PR, Castro MG. Viral vectors for gene delivery and gene therapy within the endocrine system. J Endocrinol 2000;164:103-18.

56. Maurya SK, Srivastava S, Joshi RK. Retroviral vectors and gene therapy: an update. Indian J Biotechnol 2009;8:349-57.

57. Vigna E, Naldini L. Lentiviral vectors: excellent tools for experimental gene transfer and promising candidates for gene therapy. J Gene Med 2000;2:308-16.

58. Escors D, Breckpot K. Lentiviral vectors in gene therapy: their current status and future potential. Arch Immunol Ther Exp 2010;58:107-19.

59. Cockrell AS, Kafri T. Gene delivery by lentivirus vectors. Mol Biotechnol 2007;36:184-204.

60. C Silva A, M Lopes C, M Sousa Lobo J, Helena Amaral M. Nucleic acids delivery systems: a challenge for pharmaceutical technologists. Curr Drug Metab 2015;16:3-16.

61. Volpers C, Kochanek S. Adenoviral vectors for gene transfer and therapy. J Gene Med 2004;6(S1):S164-71.

62. Gill DR, Davies LA, Pringle IA, Hyde SC. The development of gene therapy for diseases of the lung. Cell Mol Life Sci 2004;61:355-68

63. Kootstra NA, Verma IM. Gene therapy with viral vectors. Annu Rev Pharmacol Toxicol 2003;43:413-39.

64. Manservigi R, Argnani R, Marconi P. HSV recombinant vectors for gene therapy. Open Virol J 2010;4:123

65. Vannucci L, Lai M, Chiuppesi F, Ceccherini-Nelli L, Pistello M. Viral vectors: a look back and ahead on gene transfer technology. New Microbiol 2013;36:1-22.

66. Kanesa Thasan N, Smucny JJ, HokeJr CH, Marks DH, Konishi E Kurane I, et al. Safety and immunogenicity of NYVAC-JEV and ALVAC-JEV attenuated recombinant Japanese encephalitis virus-poxvirus vaccines in vaccinia-nonimmune and vacciniaimmune humans. Vaccine 2000;19:483-91. 
67. Doehn C, Kausch I, Boehmer T, Sommerauer M, Jocham D. Drug evaluation: Therion'srV-PSA-TRICOM+rF-PSA-TRICOM primeboost prostate cancer vaccine. Curr Opin Mol Ther 2007;9:183.

68. Kirn DH, Thorne SH. Targeted and armed oncolytic poxviruses: a novel multi-mechanistic therapeutic class for cancer. Nat Rev Cancer 2009;9:64-71.

69. Lam PY, Breakefield XO. Hybrid vector designs to control the delivery, fate and expression of transgenes. J Gene Med 2000;2:395-408.

70. Gonçalves MA. Adeno-associated virus: from defective virus to effective vector. Virol J 2005;2:43.

71. Reynolds PN, Feng M, Curiel DT. Chimeric viral vectors the best of both worlds? Mol Med Today 1999;5:25-31.
72. Fraefel C, Jacoby DR, Lage C, Hilderbrand H, Chou JY, Alt FW, Breakefield XO, et al. Gene transfer into hepatocytes mediated by helper virus-free HSV/AAV hybrid vectors. Mol Med 1997;3:813-25.

73. Costantini LC, Wang S, Fraefel C, Breakefield XO, Isacson O. Gene transfer to the nigrostriatal system by hybrid herpes simplex virus/adeno-associated virus amplicon vectors. Human Gene Ther 1999;10:2481-94.

74. Hildinger M, Auricchio A, Gao G, Wang L, Chirmule N, Wilson JM. Hybrid vectors based on adeno-associated virus serotypes 2 and 5 for muscle-directed gene transfer. J Virol 2001;75:6199-203. 